\section{Case Reports in Dermatology}

\title{
Fenofibrate-Induced Lichenoid Drug Eruption: A Rare Culprit
}

\author{
Fayeza Mohammed $^{\text {a }}$ Laura L. Wally ${ }^{a}$ Jeffrey E. Karaban ${ }^{b}$ \\ Vijaya B. Reddy ${ }^{c}$ Yongsuk Lertratanakul ${ }^{d}$ \\ ${ }^{a}$ Department of Family Medicine, Presence Saint Joseph Hospital, Chicago, IL, USA; \\ ${ }^{b}$ Department of Dermatology, Presence Saint Joseph Hospital, Chicago, IL, USA; \\ 'Department of Pathology, Rush University Medical Center, Chicago, IL, USA; \\ ${ }^{\mathrm{d}}$ Department of Rheumatology, Presence Saint Joseph Hospital, Chicago, IL, USA
}

\section{Keywords}

Lichenoid · Rash · Drug eruption · Fenofibrate · Photosensitivity

\begin{abstract}
A lichenoid drug eruption is a rare side effect which can occur following the administration of several different medications. Here we describe a unique case of fenofibrate as the causative agent of a lichenoid drug eruption. This case highlights a rare and clinically significant dermatologic side effect of fenofibrate. In addition, we report a potential familial association which underscores the potential for underlying genetic mechanisms to be contributory to lichenoid drug eruptions. A reminder of the physical characteristics of lichen planus, knowledge of the temporal relation between administration of medications and a lichenoid drug eruption, recognition of the effect of UV exposure on lichenoid drug eruptions, and realization for the potential of symptoms to persist despite discontinuation of an offending agent can aid practitioners in promptly diagnosing lichenoid drug eruptions and initiating appropriate therapy.




\section{Introduction}

Lichenoid drug eruptions are a rare side effect which can occur following the administration of several types of medications. The most common manifestation includes symmetric erythematous or violaceous, flat, confluent, polygonal papules on the trunk or extremities, which resemble lichen planus [1]. Here we report a unique association between the use of fenofibrate and the development of lichenoid drug eruption.

\section{Case}

A 58-year-old Hispanic woman presented to the clinic with a 2-year history of skin changes most prominently involving the face. She described a dusky, purple skin discoloration which would worsen with exposure to sunlight. She also complained of non-pruritic dry skin in the affected areas. These skin changes were noted to occur approximately 6 months after initiation of fenofibrate for hyperlipidemia. This was the only medication she was on at the time of onset and resulted in permanent skin discoloration despite discontinuation after 1 year of usage. She denied any recent fevers, other medications, changes in diet, cosmetics, or soaps. A 12-point review of systems was positive for dry eyes but otherwise negative.

Her past medical history was significant for hyperlipidemia. Her medication history was notable for fenofibrate use as previously described. She worked as a crossing guard and denied any recent travel. Her family history was notable for rheumatoid arthritis in her mother and two brothers with a similar history of a photosensitive skin rash. One of these brothers developed purple hyperpigmentation involving his entire face with the use of atorvastatin. A second brother developed a similar rash while on statin therapy. Several of her other siblings tolerated statin therapy without development of skin changes.

Her physical examination was notable for hyperpigmentation with a purple hue involving her forehead, cheeks, and chin with sparing of the infraorbital and nasolabial skin folds (Fig. 1). Her facial skin was otherwise smooth with no discrete lesions. She was also noted to have scattered hyperpigmented macules down the lateral aspects of her neck, dorsum of her arms, and anterior tibia bilaterally (Fig. 2). The remainder of her physical exam was normal. Several topical steroid medications were prescribed without improvement including desoximetasone and clocortolone pivalate. Subsequent workup was initiated as detailed below.

Laboratory studies included a complete blood count and chemistry panel, which were significant for a mild elevation in total protein at 8.2 (6.1-8.1). Serum protein electrophoresis (SPEP) showed a mild elevation in beta 2 globulin at $0.7(0.2-0.5)$. Workup for autoimmune etiology included anti-nuclear antibody (ANA), anti-double stranded DNA antibody, anti-SSA and anti-SSB antibodies, which were all within normal limits.

A 3.5-mm punch biopsy of a right preauricular lesion was performed demonstrating a moderately dense lichenoid infiltrate of lymphocytes and scattered melanophages with separation at the dermal-epidural junction, consistent with a lichenoid dermatitis. A confirmatory punch biopsy of the left cheek performed at a later date demonstrated a slightly atrophic epidermis. An area of vacuolar alteration of the basal cell layer and lichenoid infiltrate of lymphocytes with admixed melanophages and colloid bodies was present (Fig. 3). The adjacent skin showed predominantly melanophages. The overall histologic changes were those of lichenoid interface dermatitis with postinflammatory pigmentary changes consistent with lichenoid drug eruption. Immunofluorescence stains were negative. 


\section{Discussion}

Herein we present a case of lichenoid drug eruption caused by fenofibrate. This diagnosis was made on the basis of history and physical exam findings, a temporal relationship between drug administration and symptom onset, and histopathologic features observed in her biopsy specimen. A differential diagnosis of her presentation also included several variants of lichen planus which classically affect individuals with darker complexion including lichen planus actinicus, a condition most often seen in younger individuals with Middle Eastern descent [1], and ashy dermatosis, a controversial entity characterized by blue-grey macules in otherwise healthy individuals [2]. Fenofibrate-induced photosensitivity was also considered a potential etiology in her case, though this is typically associated with more vesicular and eczematous lesions [3]. There has been one other case of lichenoid drug eruption from fenofibrate reported in the literature [4]. Furthermore, the contribution of UV radiation to lichenoid eruptions has been previously described [5].

The mechanism of lichenoid drug eruption is not fully understood; however, it is felt to be secondary to an inflammatory reaction caused by interactions between medications and immune system mediators including $\mathrm{T}$ cells and cytokines [6]. Although no genetic factors have been identified in the literature, the family history noted in the following case suggests a genetic predisposition may also play a role.

Various drugs have been associated with lichenoid drug eruption. These include but are not limited to ACE inhibitors, thiazide diuretics, antimalarials, beta-blockers, gold salts, and penicillamine [7]. Several lipid-lowering drugs have also been shown to cause lichenoid drug eruptions including statins and the fibrate gemfibrozil [8].

Skin changes have been reported several months to over a year following the first administration of the causative drug [9]. In the current case, the offending medication caused development of a rash within 6 months. Diagnosis can be made by obtaining a detailed medication history, careful evaluation of skin lesions on physical examination, obtaining a biopsy for observation of typical histopathologic characteristics including a band-like lymphocytic infiltrate at the dermal-epidermal junction [10], and resolution of skin changes following discontinuation of an offending agent. In some cases, the eruption can recur and remit even though the causative agent is discontinued, as occurred in the current case [11,12].

Regarding treatment, most lichenoid drug eruptions self-resolve weeks to months after discontinuation of the medication. In some patients, the drug eruption can recur despite stopping the offending agent, in which case therapy may be required. Additional clinical scenarios which may warrant further treatment include prolonged symptoms impacting quality of life such as pruritis. Topical steroids such as clobetasol or fluocinonide can be used; however, they may not offer relief in some patients as in the current case. For patients with extensive disease or those who do not respond to topical steroids, oral corticosteroids such as prednisone may be required [13].

Evaluation of skin lesions by physicians requires careful attention to the history and physical examination including a detailed medication history. A reminder of the physical characteristics of lichen planus, knowledge of the temporal relation between administration of medications and a lichenoid drug eruption, recognition of the effect of UV exposure on lichenoid drug eruptions, and realization for the potential of symptoms to persist despite discontinuation of an offending agent can aid practitioners in promptly diagnosing lichenoid drug eruptions and initiating appropriate therapy. 


\section{Statement of Ethics}

Informed consent was obtained from our patient in accordance to institutional guidelines.

\section{Disclosure Statement}

The authors have no financial conflicts of interest to disclose.

\section{References}

1 Meads SB, et al: Lichen planus actinicus. Cutis 2003;72:377-381.

-2 Vega ME, et al: Ashy dermatosis and lichen planus pigmentosus: a clinicopathologic study of 31 cases. Int J Dermatol 1992;31:90-94.

-3 Serrano G, et al: Photosensitivity induced by fibric acid derivatives and its relation to photocontact dermatitis to ketoprofen. J Am Acad Dermatol 1992;27:204-208.

4 Gardeazabal J, Gonzalez M, Izu R, Gil N, Aguirre A, Diaz-Perez JL: Phenofibrate-induced lichenoid photodermatitis. Photodermatol Photoimmunol Photomed 1993;9:156-158.

-5 Stein KR, Scheinfeld NS: Drug-induced photoallergic and phototoxic reactions. Expert Opin Drug Saf 2007;6:431.

-6 Yawalkar N, Pichler WJ: Mechanisms of cutaneous drug reactions (in German). J Dtsch Dermatol Ges 2004;2:1013-1023.

7 Halevy S, Shai A: Lichenoid drug eruptions. J Am Acad Dermatol 1993;29:249.

8 Bork K: Lichenoid eruptions. In: Bork K (ed): Cutaneous Side Effects of Drugs. WB Saunders, Philadelphia, 1988, p 170.

9 Brauer J, Votava HJ, Meehan S, Soter NA: Lichenoid drug eruption. Dermatol Online J 2009;15:13.

10 Lage D, Juliano PB, Metze K, et al: Lichen planus and lichenoid drug-induced eruption: a histological and immunohistochemical study. Int J Dermatol 2012;51:1199.

11 Anderson TE: Lichen planus following quinidine therapy. Br J Dermatol 1967;79:500.

12 Seehafer JR, Rogers RS 3rd, Fleming CR, Dickson ER: Lichen planus-like lesions caused by penicillamine in primary biliary cirrhosis. Arch Dermatol 1981;117:140.

13 Cribier B, Frances C, Chosidow 0: Treatment of lichen planus. An evidence-based medicine analysis of efficacy. Arch Dermatol 1998;134:1521. 


\section{Case Reports in

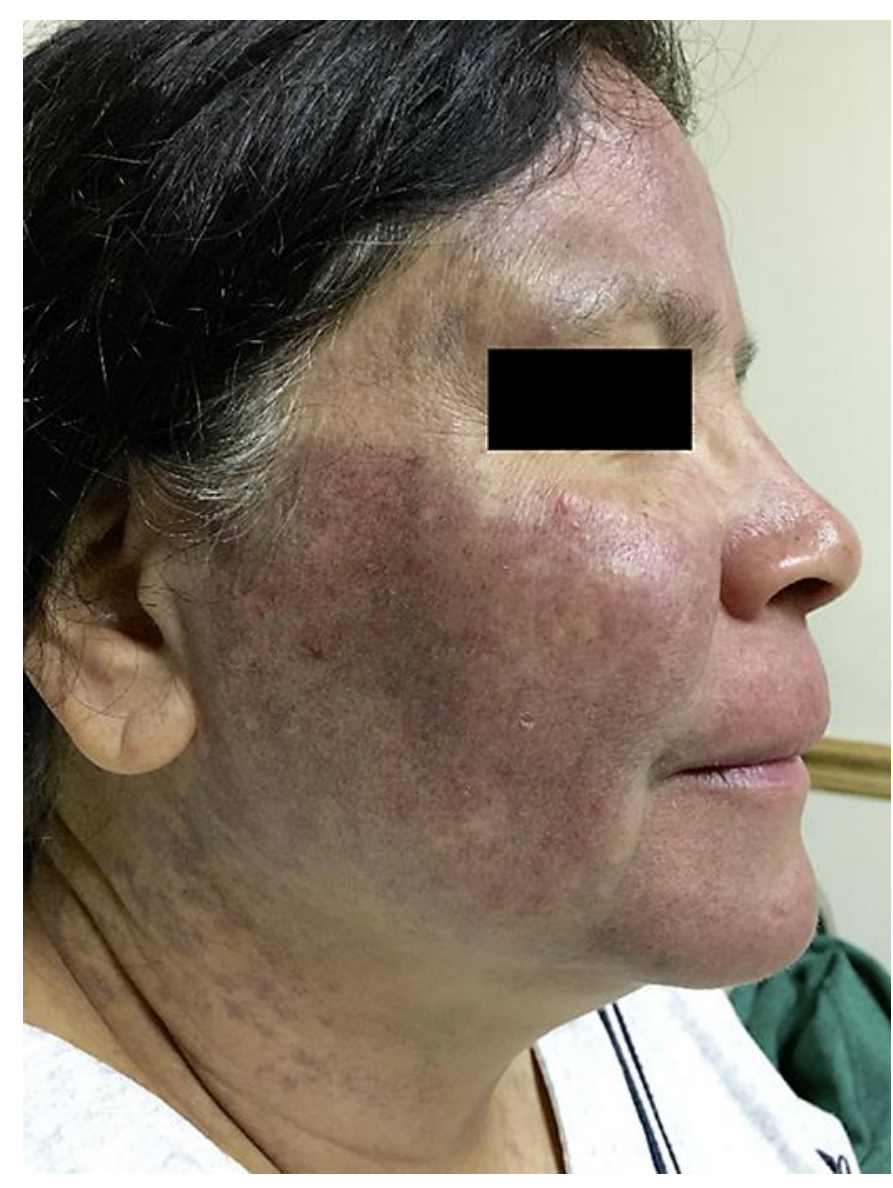

Fig. 1. Lichenoid drug eruption with postinflammatory hyperpigmentation involving the entire face with sparing of infraorbital and nasolabial skin folds. 


\section{Case Reports in \\ Dermatology}

Case Rep Dermatol 2017;9:236-242

(C) 2017 The Author(s). Published by S. Karger AG, Basel www.karger.com/cde

Mohammed et al.: Fenofibrate-Induced Lichenoid Drug Eruption: A Rare Culprit

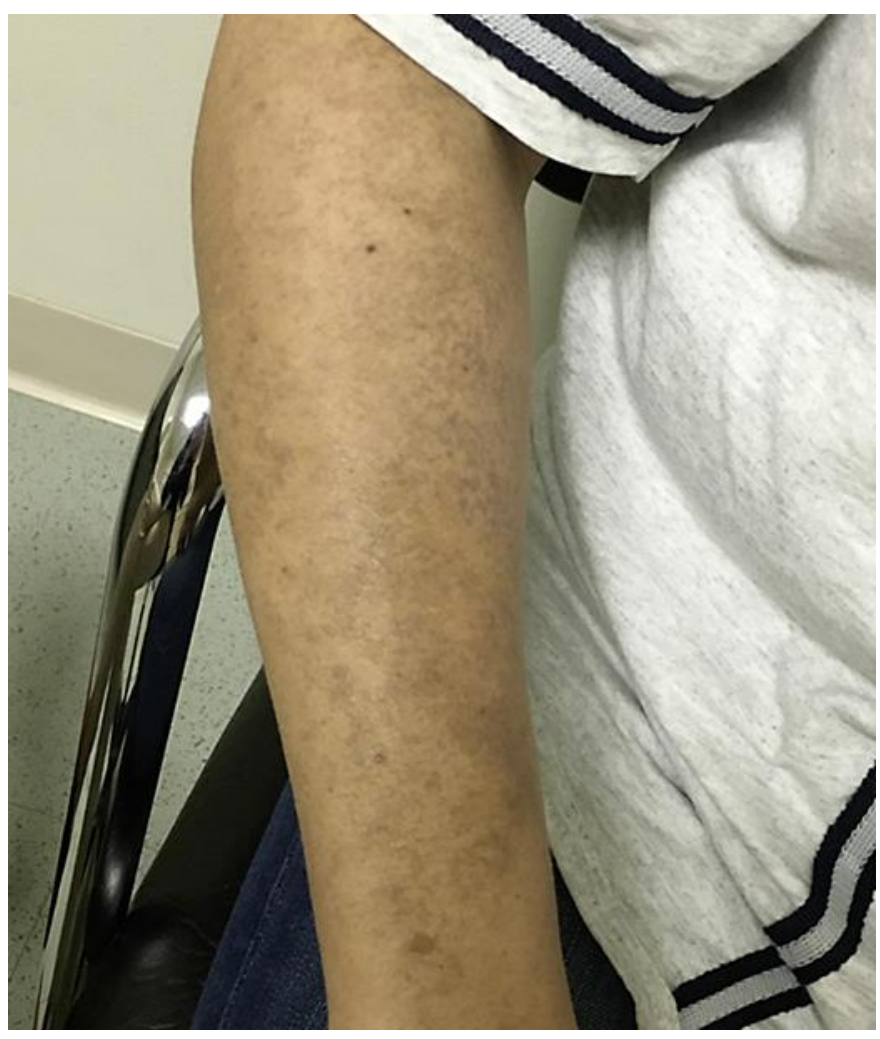

Fig. 2. Extensor surface of right arm demonstrating confluent violaceous lichenoid drug eruption and residual hyperpigmentation. 


\section{Case Reports in \\ Dermatology}

Case Rep Dermatol 2017;9:236-242

(c) 2017 The Author(s). Published by S. Karger AG, Basel www.karger.com/cde

Mohammed et al.: Fenofibrate-Induced Lichenoid Drug Eruption: A Rare Culprit

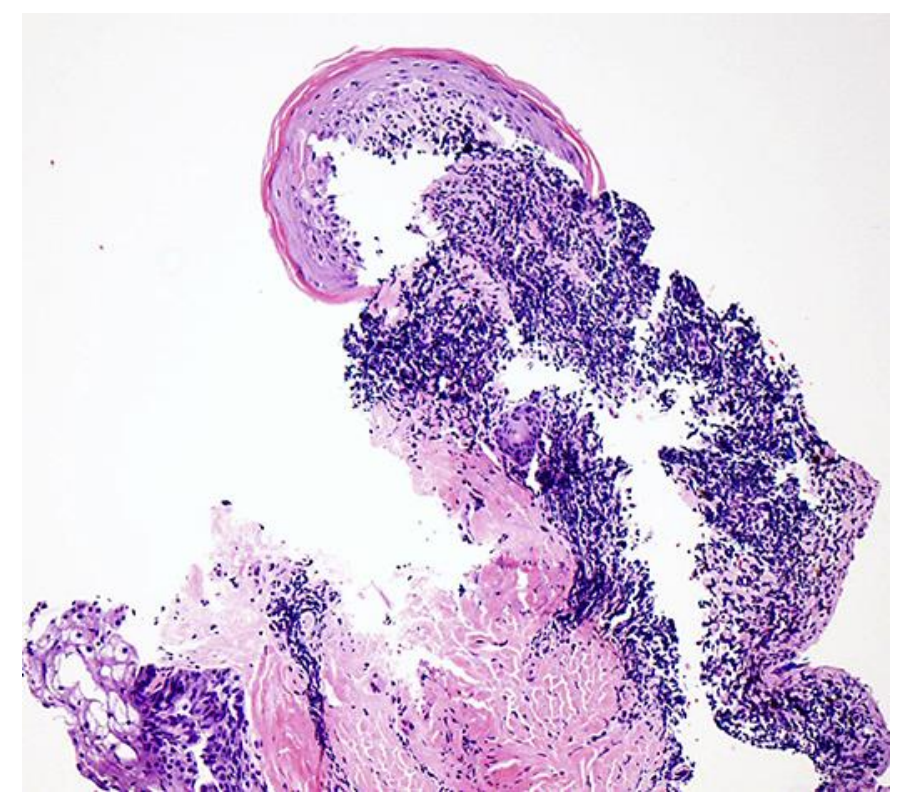

Fig. 3. Punch biopsy of the left cheek demonstrating slightly atrophic epidermis with an area of vacuolar alteration of the basal cell layer and lichenoid infiltrate of lymphocytes with admixed melanophages and colloid bodies. 